\section{RMD Open}

Rheumatic \&

Musculoskeletal Diseases

\title{
Latent profile analysis approach to the relationship between patient and physician global assessments of rheumatoid arthritis activity
}

\author{
George A Karpouzas, ${ }^{1}$ Vibeke Strand, ${ }^{2}$ Sarah R Ormseth ${ }^{1}$
}

To cite: Karpouzas GA, Strand V, Ormseth SR. Latent profile analysis approach to the relationship between patient and physician global assessments of rheumatoid arthritis activity. RMD Open 2018;4:e000695. doi:10.1136/

rmdopen-2018-000695

- Prepublication history and additional material for this paper are available online. To view these files, please visit the journal online (http://dx.doi. org/10.1136/rmdopen-2018000695).

Received 5 April 2018 Revised 6 June 2018 Accepted 11 June 2018

Check for updates

(C) Author(s) (or their employer(s)) 2018. Re-use permitted under CC BY-NC. No commercial re-use. See rights and permissions. Published by BMJ.

${ }^{1}$ Division of Rheumatology, Harbor-UCLA Medical Center and Los Angeles Biomedical Research Institute, Torrance, California, USA

${ }^{2}$ Division of Immunology/ Rheumatology, Stanford University School of Medicine, Palo Alto, California, USA

Correspondence to Dr George A Karpouzas; gkarpouzas@labiomed.org

\section{ABSTRACT}

Objective Patients and physicians commonly differ in their assessments of rheumatoid arthritis (RA) activity. Clinically meaningful discordance thresholds or validation of their ability to predict functional outcomes are lacking. We explored whether an unbiased, person-centred latent profile analysis (LPA) approach could classify cases based on patient global assessment (PtGA) and physician global assessment (MDGA) assessments of RA activity. We further examined whether the LPA groups displayed greater differences in clinical outcomes compared with traditional threshold-based groups. Finally, we evaluated whether LPA yielded higher explanatory power for clinical outcomes. Methods LPA was performed in 618 patients with established RA from a single centre. A threshold-based discordance definition was used as a comparator, with patients classified into concordant (PtGA-MDGA within $\pm 3 \mathrm{~cm}$ ), positively discordant (PtGA-MDGA $\geq 3 \mathrm{~cm}$ ) and negatively discordant groups (PtGA-MDGA $\leq-3 \mathrm{~cm}$ ). Results LPA yielded five distinct groups: low PtGA/ Iow MDGA (35.9\%), moderate PtGA/moderate MDGA (18.6\%), high PtGA/high MDGA (14.7\%), high PtGA/low MDGA (23.3\%) and low PtGA/high MDGA (7.4\%). Groups differed across clinical, physical function, pain, fatigue, health-related quality of life, work productivity and activity impairment outcomes $(p<0.001)$. Concordance groups, in particular, displayed marked heterogeneity in outcomes depending on the magnitude of disease activity reported, with the low/low group faring the best $(p<0.001)$. The LPA solution demonstrated superior explanatory power for all outcomes $(p<0.001)$.

Conclusions We confirmed the validity and advantages of LPA in characterising the relationship between PtGA and MDGA over a conventional threshold-based definition. LPA yielded optimally distinct, clinically meaningful and cohesive groupings, demonstrating superior explanatory power for disease-related outcomes of interest.

\section{INTRODUCTION}

Patients and physicians commonly differ in their evaluations of rheumatoid arthritis (RA) activity. ${ }^{1-4}$ Such discrepancies have been traditionally reported as a difference or discordance score by subtracting physician global
Key Messages

What is already known about this subject?

- Patients and physicians commonly differ in their evaluations of rheumatoid arthritis activity. Clinically meaningful discordance thresholds have not been empirically derived, and the ability of the ones used to optimally differentiate clinical, functional and health-related quality of life (HRQOL) outcomes has not been adequately explored.

What does this study add?

- An unbiased, empirically derived, patient-centred latent profile analysis approach to characterise the relationship between patient and physician assessments of disease activity identified diverse and clinically meaningful patient groups.

- This five-profile solution was numerically distinct from the traditional three-group, threshold-based discordance approach and demonstrated greater predictive power for disease-related, clinical, functional and $\mathrm{HRQOL}$ outcomes.

How might this impact on clinical practice?

- Our findings suggest that simply considering the magnitude of disagreement between patients and physicians is less clinically useful than carefully contemplating the reporting pattern (ie, which informant reports greater disease activity) and whether patients and physicians agree or disagree on the presence of high or low disease activity levels.

assessment (MDGA) from patient global assessment (PtGA). ${ }^{1245}$ Based on this, patients are arbitrarily classified as concordant, positively discordant (higher patient activity) or negatively discordant (higher physician activity). Despite this being intuitively appealing and computationally convenient, there are neither empirical evidence nor strong a priori hypotheses supporting the number or nature of the groups that qualify the relationship between PtGA and MDGA. Both are integral components of the American College of 
Rheumatology (ACR) core set and (some) remission definitions and further inform therapeutic decisions. ${ }^{67}$ Since determinants of those scores and their relative contributions are vastly disparate, the concept of a discrepancy score generated by simple subtraction of two heterogeneous constructs as a predictor of functional outcomes is theoretically and statistically problematic. ${ }^{8}$ Moreover, the ability of the various, widely reported arbitrary cut-offs to optimally differentiate clinical, functional and health-related quality of life (HRQoL) outcomes between patient groups has not been explored. Additionally, the magnitude of RA activity patients and physicians consensually report is not taken into consideration in the conventional framework of a difference score. It has been shown that when RA is in remission, patients enjoy superior physical function and incur less radiographic progression than when it is highly active. ${ }^{9}$

Another concern is that studies evaluating the impact of patient-physician discordance on various outcomes of interest have used variable-centred methodologies such as multiple regression ${ }^{14}$; such approaches assume that the entire population is homogeneous and therefore results reflect the relationships averaged over the entire population. Additionally, since they focus on the structure of the variables across persons, rather than the patterns of response within persons,${ }^{10}$ they may be inappropriate when seeking to interpret individual data. ${ }^{11}$ In contrast, person-centred approaches assume that the population is heterogeneous and allow for examination of patterns and relationships among variables at the individual level. ${ }^{11-13}$

Latent profile analysis (LPA) is an empirically derived, person-centred approach that focuses on relations among individuals with the purpose of sorting them into groups of subjects who are similar to each other and different from the other groups. It identifies the smallest number of latent groups required to account for the distribution of individuals across indicators. ${ }^{14} 15$ The number of groups that best fits the data is determined statistically rather than subjectively. ${ }^{16}$ The purpose of our study was to evaluate the ability of LPA to classify cases based on the patterns of relationships between PtGA and MDGA. We then examined the potential overlap and discrepancies between the latent profiles and traditional discordance groups and compared their respective explanatory power for outcomes of interest. We lastly assessed the concurrent validity of the LPA-generated groups by exploring associations between the profiles and clinical, functional and HRQoL outcomes.

\section{METHODS}

\section{Study design and participants}

Study participants were enrolled in the Harbor-University of California Los Angeles (UCLA) prospective observational RA cohort between 2012 and 2017. ${ }^{17}$ The first visit with complete data available for all predictors and outcomes of interest within that time frame was selected for analysis. We evaluated 618 patients with established RA. Patients were included in the study if they were $\geq 18$ years old and fulfilled 2010 ACR criteria for RA. ${ }^{18}$ Patients with overlapping autoimmune syndromes or comorbid conditions that could confound RA treatments (including chronic infections, advanced or decompensated heart failure, class II chronic kidney disease or above and cancer within 5 years) or at risk of suicide were excluded. All patients provided written informed consent in compliance with the Helsinki Declaration, and the study was approved by the Harbor-UCLA Institutional Review Board.

\section{Measures}

PtGA and MDGA ratings were recorded on $10 \mathrm{~cm}$ visual analogue scale (VAS) anchored by 'very good' or 'no activity' on the left and 'very bad' or 'high activity' on the right, respectively. Prior to the visit, patients completed self-report questionnaires; the PtGA question asked was 'Considering all the ways your arthritis has affected you, how do you feel your arthritis is today?'. This was in accordance to the proposed phrasing by the ACR/European Leagues Against Rheumatology remission criteria related to disease activity. ${ }^{1920}$ After conclusion of the index visit, the examining physician (blinded to the patient's rating) recorded their activity evaluation (MDGA) based on history, physical examination and available laboratory tests. The prompt given to the physician was 'Mark on the line below to indicate disease activity (independent of the patient's self-assessment)'. The conventional discordance definition comparator included a PtGA-MDGA difference score of $\geq 3$, informed by a recent meta-analysis indicating this to be the most frequently used threshold (5); based on this cut-off, patients were classified into three groups: concordant (PtGA-MDGA within $\pm 3 \mathrm{~cm}$ ), positively discordant (PtGA-MDGA $\geq 3 \mathrm{~cm}$ ) and negatively discordant (PtGA-MDGA $\leq-3 \mathrm{~cm})$.

Demographic, clinical, serological, laboratory, radiographic and treatment data were obtained via chart review. Disease activity assessment was based on 28-joint counts for tenderness joint count, swelling joint coun and erythrocyte sedimentation rate (ESR). Presence of erosions and irreversible articular damage (IAD) defined as subluxation, fusion, contracture or fixed deformity, arthrodesis or prosthesis were recorded. The presence of fibromyalgia based on the ACR preliminary classification criteria ${ }^{21}$ was also captured.

Physical function was evaluated using the Health Assessment Questionnaire Disability Index (HAQ-DI). ${ }^{22}$ The nine-item Patient Health Questionnaire (PHQ-9) 2324 assessed depressive symptoms (range 0-27). Pain and fatigue were both measured using $10 \mathrm{~cm}$ VAS. HRQoL was evaluated by the physical component summary and mental component summary and eight domain scores of the 36-item Short Form Survey (SF-36). ${ }^{25}$ The Work Productivity and Activity Impairment Questionnaire ${ }^{26}$ assessed activity impairment due to RA and percentage of work productivity loss among employed subjects. 


\section{Statistical analysis}

We performed LPA using the Mplus software package (Muthen \& Muthen). LPA models with increasing number of groups were fit to the data. The optimal number of profiles was determined based on the Bayesian information criterion, the Vuong-Lo-Mendell-Rubin likelihood ratio test, the Akaike information criterion and entropy. ${ }^{142728}$ An a priori decision was made to abort testing models with increasing group numbers if at least two fit statistics suggested no further improvement. The Mplus output includes scores for the conditional probability of each patient being a member of any of the LPA groups, allowing evaluation of how well the model classifies patients. ${ }^{29}$ For the purposes of group comparison and external validation, we assigned patients to the profile for which they had the highest conditional probability. Latent profiles were related to demographic and clinical characteristics with analysis of variance (ANOVA) or $\chi^{2}$ tests. The meaningfulness of the LPA solution was assessed using analysis of covariance, which evaluated between-group differences on clinical, functional and HRQoL outcomes. We used adjusted $\Delta R^{2}$ value (per cent unique variance explained) to assess the incremental explanatory power of regression models from the LPA-derived solution compared with the traditional discordance group definition to predict outcomes of interest after the effects of relevant clinical covariates had been removed. Age, gender, RA duration, IAD and fibromyalgia were included as descriptive clinical variables in all covariate adjusted analyses. Significance level was set at $\mathrm{p}<0.05$, and post hoc pairwise comparisons were Benjamini-Hochberg corrected. ${ }^{30}$ Analyses were performed using SPSS V.21.
RESULTS

\section{LPA identifies five distinct patient groups}

A five-group solution provided the best fit for the data (online supplementary table 1). The five distinct patient groups based on different pairings of PtGA and MDGA appear in figure 1: group 1: low PtGA/low MDGA $(\mathrm{n}=222,35.9 \%)$; group 2: moderate $\mathrm{PtGA} /$ moderate MDGA ( $\mathrm{n}=115,18.6 \%)$; group 3: high PtGA/high MDGA $(\mathrm{n}=91,14.7 \%)$; group 4: high PtGA/low MDGA $(\mathrm{n}=144$, 23.3\%); and group 5: low PtGA/high MDGA $(n=46$, $7.4 \%)$. Demographics and clinical characteristics for all groups are summarised in table 1 below.

Patients were largely female with established, robustly seropositive and erosive RA. Seropositivity, radiographic erosions and treatment characteristics were similarly distributed across all LPA groups. Group 4 members were older, with longer disease duration and higher fibromyalgia rates compared with other groups (all $\mathrm{p} \leq 0.01)$. Clinical and serological inflammatory burden was significantly different across groups $(\mathrm{p}<0.001)$.

\section{Comparisons of LPA and traditional discordance groups}

The PtGA and MDGA distributions across the three traditional threshold-based groups (negatively discordant, concordant and positively discordant versus those in the LPA-generated class definition are shown in online supplementary figure 1 . Similar to the traditional concordance category, patients in LPA groups 1, 2 and 3 displayed congruent PtGA and MDGA scores; however, this congruence referred to three very different inflammatory activity states. In group 1, both parties reported low disease activity (mean PtGA and MDGA of 1.9 and

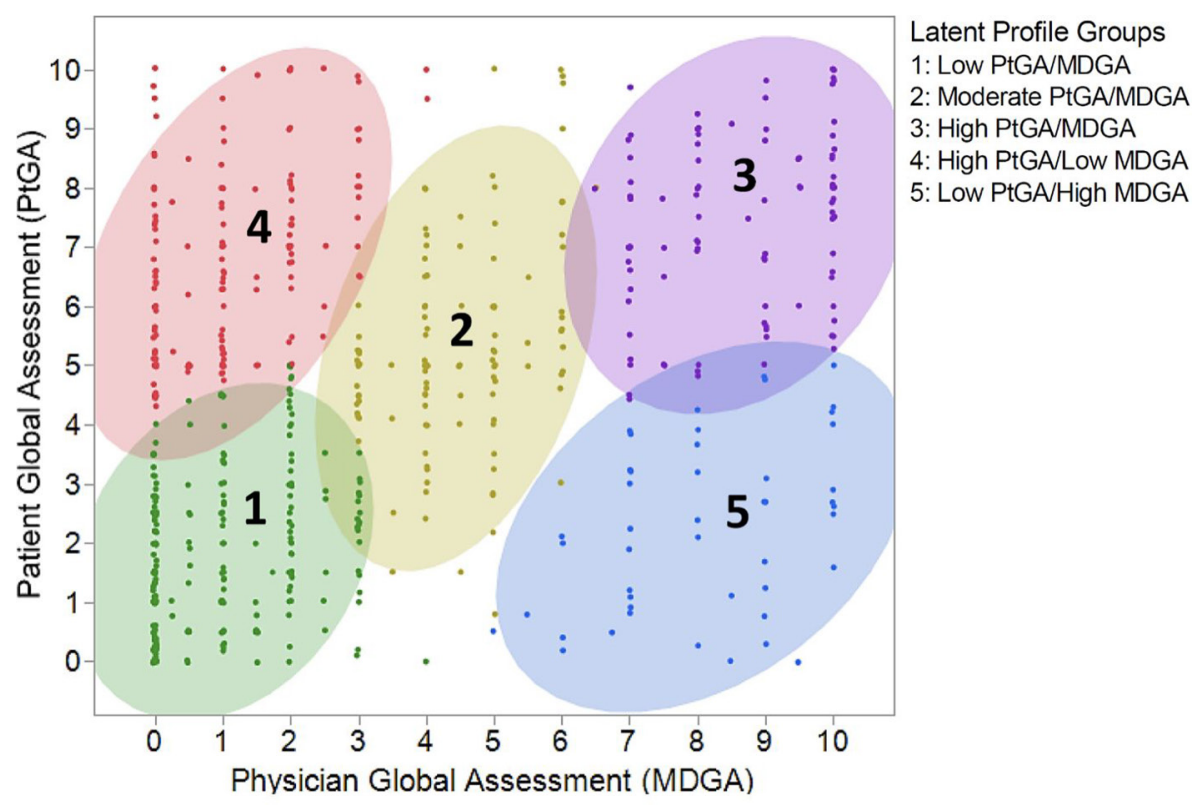

Figure 1 LPA identified five distinct patient groups based on different pairings of PtGA and MDGA. Group 1: low PtGA/low MDGA ( $n=222,35.9 \%)$, group 2: moderate PtGA/moderate MDGA $(n=115,18.6 \%)$, group 3: high PtGA/high MDGA ( $n=91$, 14.7\%), group 4: high PtGA/low MDGA ( $n=144,23.3 \%)$ and group 5: low PtGA/high MDGA ( $n=46$, 7.4\%). LPA, latent profile analysis; PtGA, patient global assessment of disease activity; MDGA, physician global assessment of disease activity. 
Table 1 Demographic and clinical characteristics for latent profile groups

\begin{tabular}{|c|c|c|c|c|c|c|}
\hline & $\begin{array}{l}\text { Low PtGA/low } \\
\text { MDGA }(n=222)\end{array}$ & $\begin{array}{l}\text { Moderate PtGA/ } \\
\text { moderate MDGA } \\
(n=115)\end{array}$ & $\begin{array}{l}\text { High PtGA/high } \\
\text { MDGA }(n=91)\end{array}$ & $\begin{array}{l}\text { High PtGA/low } \\
\text { MDGA }(n=144)\end{array}$ & $\begin{array}{l}\text { Low PtGA/high } \\
\text { MDGA }(n=46)\end{array}$ & $P$ values \\
\hline Age, years & $52.4 \pm 12.1_{a}$ & $49.9 \pm 11.0_{a}$ & $50.8 \pm 10.9_{a}$ & $55.4 \pm 11.1_{b}$ & $49.8 \pm 10.9_{a}$ & 0.001 \\
\hline Sex (female) & $186(83.8)$ & $99(86.1)$ & $82(90.1)$ & $131(91.0)$ & $39(84.8)$ & 0.28 \\
\hline RA duration, years & $10.5 \pm 7.9_{a}$ & $9.2 \pm 7.3 \mathrm{a}$ & $10.2 \pm 7.6_{\mathrm{ab}}$ & $12.8 \pm 9.8_{b}$ & $9.1 \pm 9.1_{\mathrm{a}}$ & 0.01 \\
\hline RF positive & 207 (93.2) & $106(92.2)$ & 83 (91.2) & $128(88.9)$ & 42 (91.3) & 0.58 \\
\hline ACPA positive & $197(88.7)$ & $101(87.8)$ & $81(89.0)$ & $124(86.1)$ & 42 (91.3) & 0.88 \\
\hline Erosions & $110(49.5)$ & $54(47.0)$ & $44(48.4)$ & $84(58.3)$ & $24(52.2)$ & 0.54 \\
\hline IAD present & $53(23.9)$ & $34(29.6)$ & $26(28.6)$ & $46(31.9)$ & $9(19.6)$ & 0.33 \\
\hline Fibromyalgia & $24(10.8)_{a}$ & $16(13.9)_{\mathrm{ab}}$ & $15(16.5)_{\mathrm{ab}}$ & $36(25.0)_{b}$ & $5(10.9)_{a b}$ & 0.01 \\
\hline $\begin{array}{l}\text { Number of } \\
\text { csDMARDs }\end{array}$ & $1.6 \pm 0.9$ & $1.4 \pm 0.9$ & $1.3 \pm 1.1$ & $1.5 \pm 1.0$ & $1.6 \pm 1.0$ & 0.27 \\
\hline bDMARD use & $74(33.3)$ & $42(36.5)$ & 33 (36.3) & $50(34.7)$ & 15 (32.6) & 0.97 \\
\hline SJC (28) & $1.1 \pm 1.5_{\mathrm{a}}$ & $4.4 \pm 2.3_{b}$ & $10.5 \pm 4.7_{c}$ & $1.0 \pm 1.3_{a}$ & $9.9 \pm 4.2_{c}$ & $<0.001$ \\
\hline TJC (28) & $1.2 \pm 2.5_{a}$ & $5.9 \pm 3.9_{b}$ & $12.9 \pm 6.5_{c}$ & $2.0 \pm 3.7_{a}$ & $9.9 \pm 6.2_{d}$ & $<0.001$ \\
\hline ESR (mm/hour) & $28.0 \pm 17.2_{a}$ & $36.8 \pm 22.2_{b}$ & $52.2 \pm 26.1_{c}$ & $31.1 \pm 19.2_{a}$ & $42.7 \pm 21.7_{b}$ & $<0.001$ \\
\hline $\mathrm{CRP}(\mathrm{mg} / \mathrm{dL})$ & $0.6 \pm 0.7 \mathrm{a}$ & $1.3 \pm 1.8_{\mathrm{bd}}$ & $2.6 \pm 3.2_{c}$ & $0.9 \pm 1.0_{\mathrm{ab}}$ & $1.7 \pm 2.9_{d}$ & $<0.001$ \\
\hline DAS28-ESR & $3.0 \pm 0.9$ & $5.0 \pm 0.7 \mathrm{~b}$ & $6.5 \pm 0.8_{c}$ & $3.9 \pm 0.9_{d}$ & $5.4 \pm 0.8_{e}$ & $<0.001$ \\
\hline PtGA & $1.9 \pm 1.3_{a}$ & $5.3 \pm 1.8_{b}$ & $7.4 \pm 1.5_{c}$ & $6.9 \pm 1.6_{d}$ & $2.3 \pm 1.5_{a}$ & $<0.001$ \\
\hline MDGA & $1.0 \pm 1.0_{\mathrm{a}}$ & $4.4 \pm 1.0_{b}$ & $8.6 \pm 1.2_{c}$ & $1.2 \pm 1.1_{\mathrm{a}}$ & $8.1 \pm 1.4_{d}$ & $<0.001$ \\
\hline $\begin{array}{l}\text { PtGA-MDGA } \\
\text { difference }\end{array}$ & $0.8 \pm 1.5$ & $0.8 \pm 1.7_{a}$ & $-1.2 \pm 1.7_{b}$ & $5.7 \pm 1.5_{c}$ & $-5.8 \pm 1.6_{d}$ & $<0.001$ \\
\hline
\end{tabular}

Values are the mean \pm SD or number (per cent). Group comparisons made using $\chi^{2}$ and analysis of variance tests for categorical and continuous variables. $\mathrm{P}$ values for pairwise contrasts are Benjamini-Hochberg adjusted; values in a row not sharing subscript letters (a, $b$ and c) denote subgroups whose averages differ significantly $(p<0.05)$.

ACPA, anticyclic citrullinated peptide antibodies; bDMARDs, biological disease modifying antirheumatic drugs; CRP, $\mathrm{C}$ reactive protein; DAS28-ESR, 28 joint-based disease activity index with ESR; ESR, erythrocyte sedimentation rate; IAD, irreversible articular damage; MDGA, physician global assessment; n-csDMARDs, number of concurrent conventional synthetic disease modifying antirheumatic drugs; PtGA, patient global assessment; RA, rheumatoid arthritis; RF, rheumatoid factor; SJC, swelling joint count out of 28 joints; TJC, tenderness joint count out of 28 joints.

1.0, respectively), in group 2: moderate disease activity (5.3 and 4.4, respectively) and in group 3: high disease activity (7.4 and 8.6, respectively). A cross-tabulation of LPA-derived and the traditional discordance groups appears in table 2.

This revealed that patients assigned to positive and negative discordance groups using the conventional definition were, for the most part, members of a latent profile with comparable PtGA-MDGA differences: high PtGA/low MDGA (group 4) and low PtGA/high MDGA (group 5), respectively. In contrast, the traditional concordance category was more of a true amalgam of members from the low PtGA/low MDGA group 1 (54.4\%), moderate PtGA/moderate MDGA group 2

Table 2 Cross-tabulation of latent profile and traditional discordance groups

\begin{tabular}{lccc}
\hline & \multicolumn{2}{l}{ Traditional discordance categories } & \\
\cline { 2 - 4 } Latent profiles & Negative discordance & Concordance & Positive discordance \\
\hline Low PtGA/low MDGA & $1(1.5 \%)$ & $203(54.4 \%)$ & $18(10.2 \%)$ \\
Moderate PtGA/moderate MDGA & $3(4.4 \%)$ & $97(26.0 \%)$ & $15(8.5 \%)$ \\
High PtGA/high MDGA & $18(26.5 \%)$ & $73(19.6 \%)$ & 0 \\
High PtGA/low MDGA & 0 & 0 & $144(81.4 \%)$ \\
Low PtGA/high MDGA & $46(67.6 \%)$ & 0 & 0 \\
Total & $68(11.0 \%)$ & $373(60.4 \%)$ & $177(28.6 \%)$ \\
\hline
\end{tabular}

All percentages are column percentages.

MDGA, physician global assessment of disease activity; PtGA, patient global assessment of disease activity. 

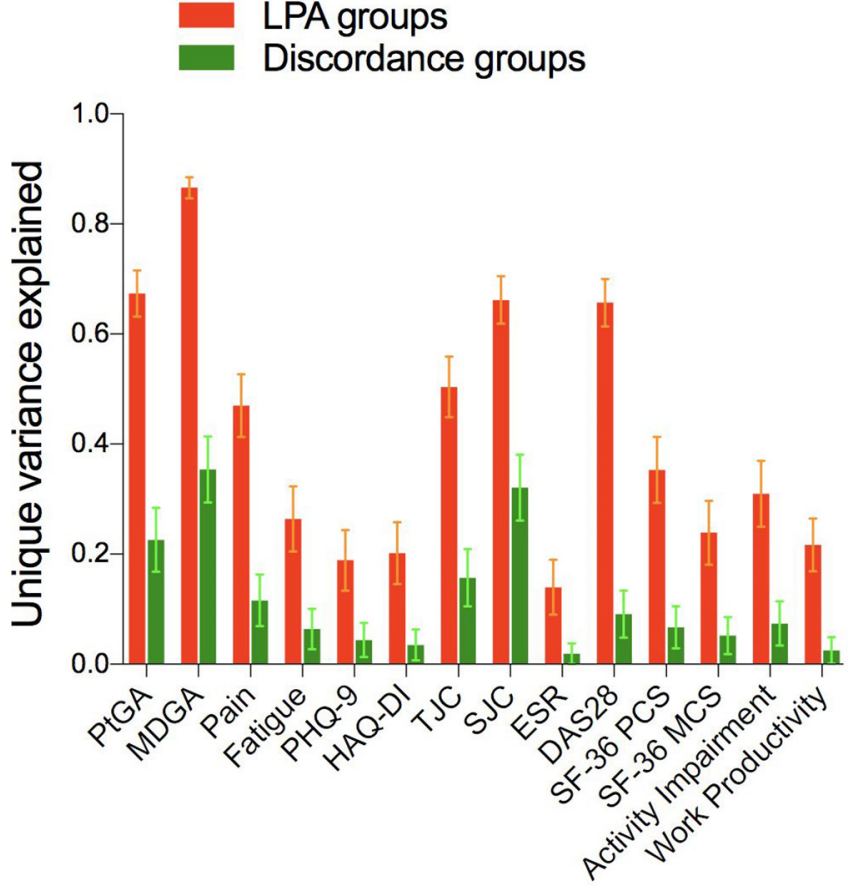

Figure 2 Explanatory power of the LPA solution for clinical and functional outcomes compared with the conventional threshold-based discordance definition. Bar height represents the unique variance accounted in each outcome by the LPA and traditional discordance approaches after controlling for the effects of age, gender, RA duration, irreversible articular damage and fibromyalgia (incremental $\triangle \mathrm{R} 2$ ). Error bars represent 95\% Cls. DAS28ESR, 28 joint-based disease activity index with ESR; ESR, erythrocyte sedimentation rate; $\mathrm{HAQ}$-DI, Health Assessment Questionnaire-Disability Index; LPA, latent profile analysis; MDGA, physician global assessment of disease activity; PHQ9, nine-item Patient Health Questionnaire; PtGA, patient global assessment of disease activity; SF36-MCS, Short Form 36 mental component score; SF36-PCS, Short Form 36 physical component score; SJC, swollen joint count; TJC, tenderness joint count.

(26.0\%) and high PtGA/high MDGA group 3 (19.6\%) latent profiles.

The LPA group solution demonstrated significantly higher explanatory power (adjusted incremental $\mathrm{R}^{2}$ ) for all inflammatory activity parameters, as well as physical function, pain, fatigue, depression, HRQoL, work productivity and activity impairment outcomes than the traditional discordance definition (figure 2 , all $\mathrm{p}<0.001$ ); this was above and beyond that accounted for by age, gender, RA duration, IAD and fibromyalgia.

\section{Clinical, functional and HRQOL outcomes}

The LPA groups differed across all clinical and laboratory evaluations of inflammatory burden (table 1). More importantly, they also reported diverse functional, HRQoL, work productivity and activity impairment outcomes as shown in figure 3 and table 3 .

This was particularly true for groups 1,2 , and 3 , which would, under usual circumstances, be collapsed into a singular traditional concordance category. Group 1 (low
PtGA/low MDGA) reported the lowest burden of physical impairment, pain, fatigue, depression, work productivity and activity impairment and enjoyed near normal physical and mental function (compared with age-matched and gender-matched norms). In contrast, group 3 (high PtGA/high MDGA) fared the worst of all groups; it reflected the highest inflammatory burden, greatest physical, mental and social function restrictions, along with worst pain, fatigue and depression scores. Group 4 (high PtGA/low MDGA) patients experienced significant RA impact despite low inflammatory burden. Surprisingly, patients in group 5 (low PtGA/high MDGA) reported significantly lower physical limitations, pain, fatigue, depression, work productivity loss and activity impairment and superior physical and mental function than group 3 subjects, despite comparable MDGA scores (table 3).

\section{DISCUSSION}

Our study demonstrates the validity and advantages of an empirically derived, person-centred approach characterising the relationship between patient and physician assessments of RA activity based on two simple measures (PtGA and MDGA); both are collected in routine practice, represent ACR core set components and inform therapeutic decisions. ${ }^{6}$

We describe several novel findings: first, LPA yielded a statistically robust five-profile solution representing distinct patient groups with clinically meaningful phenotypes. Second, the LPA-generated solution was quantitatively and qualitatively distinct from the conventional three-group discordance definition. This difference stemmed mainly from heterogeneity in the traditional concordance category regarding the magnitude of disease activity patients and physicians consensually report; despite congruent PtGA and MDGA ratings, LPA groups 1, 2, and 3 represented clearly distinct patient clusters with increasing inflammatory burden as well as progressively worse clinical, functional, HRQOL, work-related and activity impairment outcomes. These three highly disparate, empirically defined groups were essentially collapsed into a singular, conventional concordance category, which was arbitrarily defined and-by design-disregarded the absolute levels of the individual PtGA and MDGA components. Hence, the lesson learnt here was that all agreement is not created equal; rather, it is the magnitude of the disease activity that both patients and physicians concur on that largely defines functional outcomes.

Third, when both parties report high disease activity (group 3), there is objective evidence of high inflammatory burden and patients experience worse physical function, pain, fatigue, depression, HRQOL, activity impairment and work productivity than any other group. This is in agreement with reported associations of high RA inflammatory activity with worse fatigue, ${ }^{31-33}$ depression, ${ }^{34}$ pain $^{35} 36$ and physical disability. ${ }^{9}$ However, within 
A

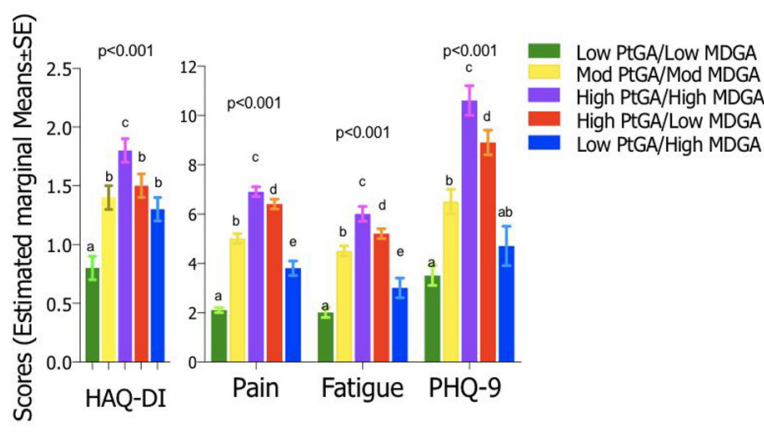

B

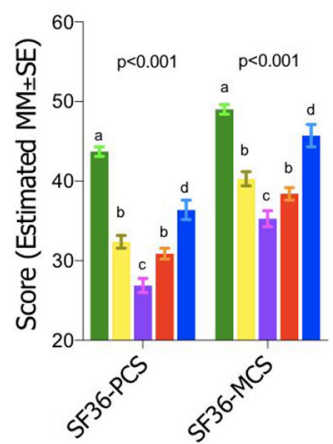

C

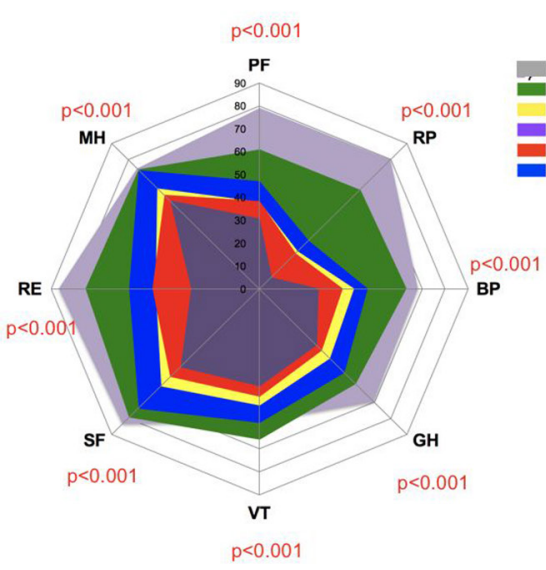

D

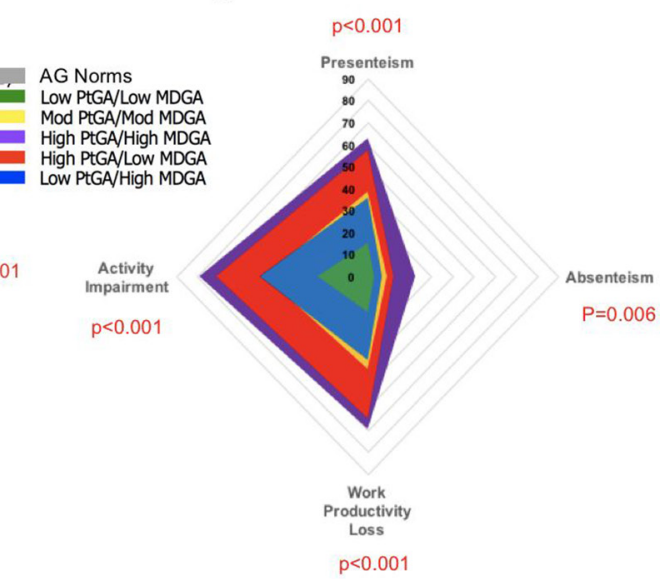

Figure 3 LPA groups display vastly different outcomes. (A) Functional disability (HAQ-DI), pain, fatigue and depression (PHQ9) scores. (B) Physical and mental component scores across LPA groups. (C) Health-related quality of life individual domain scores across LPA groups and age-matched and gender-matched controls. (D) Work productivity and activity impairment scores across LPA groups. Values represent estimated marginal means; analysis of covariance adjusted for age, gender, disease duration, fibromyalgia and presence of irreversible articular damage. All pairwise comparisons are Benjamini-Hochberg corrected. Different subscript letters denote groups that differ significantly $(p<0.05)$. All pairwise comparisons are BenjaminiHochberg-corrected; values not sharing subscript letters denote subgroups whose averages differ significantly $(p<0.05)$. BP, bodily pain; GH, general health; MDGA, physician global assessment of disease activity; MH, mental health; PF, physical function; PtGA, patient global assessment of disease activity; RE, role emotional; RP, role physical; SF, social function; SF36MCS, Short Form 36 mental component score; SF36-PCS, Short Form 36 physical component score.

the context of a traditional concordance construct, patients and physicians unanimously reported high disease activity in only a minority of cases $(73 / 373$ or $19.6 \%)$. In fact, in the majority of instances $(203 / 373$ or $54.4 \%$ ), both parties reported low or absent disease activity (group 1). As a result, the magnitude of adverse impact conferred by the high disease activity cluster 3 to the extended traditional concordance group outcomes may be grossly attenuated. Consistent with that notion, several recent studies using conventional definitions reported that patients with concordant PtGA and MDGA ratings experience better functional outcomes compared with those with positive discordance. ${ }^{43} 38$ Our results, however, clearly indicate that all such outcomes are significantly worse in group 3 that unanimously reported high disease activity compared with group 4 (high PtGA/ low MDGA), closely resembling the conventional positive discordance cluster.
Fourth, our LPA results confirmed the presence of groups qualitatively resembling the arbitrarily defined positive and negative discordance groups: latent profiles 4 and 5, respectively; this allows continued confidence in previous research derived based on such methodologies, regarding descriptions of such group characteristics and outcomes. Nevertheless, both such groups are more strictly defined by LPA; specifically, LPA group 4 represents a more homogeneous group compared with the broader traditional positive discordance cluster that is contaminated up to $18.7 \%$ by patients from groups 1 and 2, both of whom have superior outcomes. LPA group 4 patients are older, with longer disease duration and higher prevalence of fibromyalgia than all other groups. Despite minimal inflammation, indistinguishable from group 1, they experience substantial RA impact: they report significantly higher pain, fatigue and depression that directly contribute to their high PtGA scores ${ }^{4}$ and 
Table 3 Patient-reported clinical, functional and health-related quality of life outcomes for latent profiles

\begin{tabular}{|c|c|c|c|c|c|c|}
\hline All participants & $\begin{array}{l}\text { Low PtGA/low } \\
\text { MDGA }(n=222)\end{array}$ & $\begin{array}{l}\text { Moderate PtGA/ } \\
\text { moderate MDGA } \\
(n=115)\end{array}$ & $\begin{array}{l}\text { High PtGA/high } \\
\text { MDGA }(n=91)\end{array}$ & $\begin{array}{l}\text { High PtGA/low } \\
\text { MDGA }(n=144)\end{array}$ & $\begin{array}{l}\text { Low PtGA/high } \\
\text { MDGA }(n=46)\end{array}$ & $P$ values \\
\hline Pain VAS & $2.1 \pm 0.1_{a}$ & $5.0 \pm 0.2_{b}$ & $6.9 \pm 0.2_{c}$ & $6.4 \pm 0.2_{d}$ & $3.8 \pm 0.3 \mathrm{e}$ & $<0.001$ \\
\hline Fatigue VAS & $2.0 \pm 0.2 \mathrm{a}$ & $4.5 \pm 0.2_{b}$ & $6.0 \pm 0.3_{c}$ & $5.2 \pm 0.2_{d}$ & $3.0 \pm 0.4_{e}$ & $<0.001$ \\
\hline PHQ-9 & $3.5 \pm 0.4 \mathrm{a}$ & $6.5 \pm 0.5_{b}$ & $10.6 \pm 0.6_{c}$ & $8.9 \pm 0.5_{d}$ & $4.7 \pm 0.8_{\mathrm{ab}}$ & $<0.001$ \\
\hline HAQ-DI & $0.8 \pm 0.1_{\mathrm{a}}$ & $1.4 \pm 0.1_{b}$ & $1.8 \pm 0.1_{c}$ & $1.5 \pm 0.1_{b}$ & $1.3 \pm 0.1_{b}$ & $<0.001$ \\
\hline $\begin{array}{l}\text { Activity } \\
\text { impairment }\end{array}$ & $27.6 \pm 1.7_{\mathrm{a}}$ & $56.8 \pm 2.3_{b}$ & $72.8 \pm 2.6_{c}$ & $62.4 \pm 2.1_{b}$ & $43.2 \pm 3.7_{d}$ & $<0.001$ \\
\hline SF-36 PCS & $43.7 \pm 0.6_{a}$ & $32.4 \pm 0.8_{b}$ & $26.9 \pm 0.9_{c}$ & $30.9 \pm 0.7 b$ & $36.4 \pm 1.2_{d}$ & $<0.001$ \\
\hline SF-36 MCS & $49.0 \pm 0.6_{a}$ & $40.3 \pm 0.9_{b}$ & $35.3 \pm 1.0_{c}$ & $38.4 \pm 0.8_{b}$ & $45.7 \pm 1.4_{d}$ & $<0.001$ \\
\hline \multicolumn{7}{|l|}{ SF-36 domain } \\
\hline $\begin{array}{l}\text { Physical } \\
\text { functioning }\end{array}$ & $60.9 \pm 1.7_{\mathrm{a}}$ & $37.9 \pm 2.3_{b}$ & $30.8 \pm 2.6_{c}$ & $38.4 \pm 2.1_{b}$ & $47.0 \pm 3.7_{d}$ & $<0.001$ \\
\hline Role-physical & $61.3 \pm 2.3 \mathrm{a}$ & $23.3 \pm 3.3_{b}$ & $7.3 \pm 3.6_{c}$ & $22.0 \pm 3.0_{b}$ & $29.9 \pm 5.1_{b}$ & $<0.001$ \\
\hline Bodily pain & $63.4 \pm 1.3_{a}$ & $40.8 \pm 1.8_{b}$ & $25.5 \pm 2.0_{c}$ & $35.8 \pm 1.6_{d}$ & $46.8 \pm 2.8_{b}$ & $<0.001$ \\
\hline General health & $58.8 \pm 1.3_{a}$ & $43.0 \pm 1.8_{b}$ & $34.8 \pm 2.0_{c}$ & $38.0 \pm 1.6_{c}$ & $51.9 \pm 2.8_{d}$ & $<0.001$ \\
\hline Vitality & $66.0 \pm 1.2_{a}$ & $50.9 \pm 1.7_{b}$ & $42.7 \pm 1.9_{c}$ & $47.2 \pm 1.5_{b c}$ & $58.6 \pm 2.7_{d}$ & $<0.001$ \\
\hline Social functioning & $79.5 \pm 1.6_{a}$ & $60.1 \pm 2.3_{b}$ & $48.3 \pm 2.6_{c}$ & $54.4 \pm 2.1_{\mathrm{bc}}$ & $73.9 \pm 3.6_{a}$ & $<0.001$ \\
\hline Role-emotional & $75.0 \pm 2.5_{a}$ & $44.7 \pm 3.5_{b}$ & $29.6 \pm 3.9_{c}$ & $46.3 \pm 3.2_{b}$ & $56.3 \pm 5.5_{b}$ & $<0.001$ \\
\hline Mental health & $73.9 \pm 1.3_{a}$ & $62.2 \pm 1.8_{b}$ & $54.6 \pm 2.1_{c}$ & $58.0 \pm 1.7_{\mathrm{bc}}$ & $73.6 \pm 2.9_{a}$ & $<0.001$ \\
\hline $\begin{array}{l}\text { Employed } \\
\text { participants }\end{array}$ & $\begin{array}{l}\text { Low PtGA/low } \\
\text { MDGA }(n=82)\end{array}$ & $\begin{array}{l}\text { Mod PtGA/mod } \\
\text { MDGA }(n=40)\end{array}$ & $\begin{array}{l}\text { High PtGA/high } \\
\text { MDGA }(n=23)\end{array}$ & $\begin{array}{l}\text { High PtGA/low } \\
\text { MDGA }(n=24)\end{array}$ & $\begin{array}{l}\text { Low PtGA/high } \\
\text { MDGA (n=17) }\end{array}$ & $P$ values \\
\hline Absenteeism & $5.3 \pm 2.0$ & $13.0 \pm 2.8_{\mathrm{ab}}$ & $18.7 \pm 3.7_{b c}$ & $3.9 \pm 3.6_{a b}$ & $5.9 \pm 4.2_{\mathrm{abc}}$ & 0.006 \\
\hline Presenteeism & $20.9 \pm 2.8_{a}$ & $45.5 \pm 3.9_{b}$ & $59.6 \pm 5.2_{c}$ & $46.6 \pm 5.1_{\mathrm{bc}}$ & $29.4 \pm 6.0_{a}$ & $<0.001$ \\
\hline $\begin{array}{l}\text { Work productivity } \\
\text { loss }\end{array}$ & $23.9 \pm 3.0_{a}$ & $49.6 \pm 4.3_{b}$ & $66.3 \pm 5.6_{c}$ & $47.8 \pm 5.6_{b}$ & $30.0 \pm 6.5_{a}$ & $<0.001$ \\
\hline
\end{tabular}

Values represent estimated marginal means and SEs. Analysis of covariance adjusted for age, sex, disease duration, irreversible articular damage and fibromyalgia. All pairwise comparisons are Benjamini-Hochberg corrected; values in a row not sharing subscript letters denote subgroups whose averages differ significantly.

HAQ-DI, Health Assessment Questionnaire Disability Index; MCS, Mental component summary; MDGA, physician global assessment of disease activity; PCS, physical component summary; PHQ-9, nine-item Patient Health Questionnaire; PtGA, patient global assessment of disease activity; SF-36, Short Form 36 Health Survey; VAS, visual analogue scale.

yield worse functional, HRQOL and work productivity outcomes, even after controlling for age, gender, disease duration, IAD and fibromyalgia. These subjects are more poised to benefit from psychological and/or behavioural interventions to attain comprehensive remission rather than pharmacological treatments targeting RA inflammation. ${ }^{39}$ Similarly, LPA group 5 is more homogeneous compared with the extended conventional negative discordance group, which is contaminated up to $31 \%$ by group 2 and 3 patients. Specifically, LPA group 5 subjects experience significantly lower functional impairment and disease impact than expected, despite high inflammatory burden. It is possible that more adaptive illness cognitions and effective coping mechanisms ${ }^{40}{ }^{41}$ account for the significantly lower levels of pain, fatigue and depression that these patients report and contribute to their advanced social functioning, mental health, HRQOL and work productivity that resemble group 1 patients. Nevertheless, most physical function outcomes were still worse in group 5 compared with group 1 patients who were essentially at therapeutic target.

Fifth, the patient-centred LPA solution offered superior explanatory power for all disease-related parameters and outcomes of interest compared with the traditional discordance construct. This observation further supports its higher clinical relevance, validity and suitability in characterising the relationship between PtGA and MDGA. This is particularly notable since variable-oriented approaches, in general, show greater predictive power compared with person-oriented approaches. ${ }^{42}$

Our findings collectively suggest that the traditional consideration of the PtGA and MDGA relationship as a discordance or difference score (PtGA-MDGA)although intuitively appealing and computationally convenient-is arbitrary, qualitatively restrictive and methodologically suboptimal for multiple reasons. First, it is subject to all the restrictions of a variable-centred approach such as assumptions of population homogeneity 
and non-empirically derived, artificial cut-offs. Second, difference scores are less reliable than either of their component measures ${ }^{8}$; they are inherently ambiguous, as they combine into a single score constructs that are structurally and functionally disparate; they confound the effects of their component measures on outcomes and impose constraints on these effects that are rarely tested empirically ${ }^{8}$; correlations between difference scores and outcomes are often spurious as they essentially reflect the correlation of an outcome with the components from which the difference score is calculated. Since difference scores tend to correlate with either one or both of their components, the chances of observing correlations with other constructs connected to those components are amplified. Third, in the qualitatively distinct LPA solution, the number of latent groups that best fits the data is determined statistically rather than subjectively.

Hence, if one's intention is to examine the relationship between PtGA and MDGA, LPA represents a less biased and more insightful way to visualise the spatial positioning of patients across the two outcomes, beyond the problems and biases of arbitrary difference scores. However, LPA analyses may not be the most practical solution for the routine group assignment of patients based on PtGA and MDGA in daily clinical practice. The analyses are generally complex to conduct and are sample and sample size specific, in that they yield groups that may differ from study to study. The most salient point, however, is that each of the two outcomes has its own significance and therapeutic implications and therefore commands individual attention and consideration; PtGA being a broader indicator of disease impact and experience by the patient and MDGA as a barometer of inflammatory burden. We previously reported that the most significant, independent predictors of PtGA were pain $(27 \%)$, fatigue $(15 \%)$, depression $(9 \%)$, functional impairment $(8 \%)$, general health perceptions $(7 \%)$ and tender joint counts (6\%). ${ }^{4}$ Notably, improvement of PtGA over time was associated with commensurate improvements in the same exact parameters. ${ }^{4}$ Consequently, disaggregation of domains within the PtGA should be contemplated to develop management pathways targeting optimal patient-centred outcomes. ${ }^{43}$ This would be particularly true in the case of moderate or high PtGA, where illuminating the exact contribution of each of the aforementioned variables may yield a mixture of both biomedical as well as psychological/behavioural/ cognitive interventions. Future research should inform a feasible, time-efficient set of patient-reported outcomes and define how to best integrate them into daily practice. The findings should foster a standardised approach to evaluation and management, as well as improvement of patient-physician communication and shared decision making. However, we showed that MDGA bore significant correlation with composite disease activity scores such as 28 joint-based disease activity index with ESR $(r=0.85)$, and its improvement over time reflected improvement in swollen joint counts, tender joint counts, fatigue and
ESR. The implications of MDGA are, therefore, largely biomedical, an arena most rheumatologists feel more comfortable navigating.

The current work has important implications for rheumatology research: existing discordance data may be reanalysed in an effort to enrich findings already published in the literature; this may include derivation of LPA algorithms in training sets and their validation in test populations. Prevalent datasets may be combined into a unique body of evidence with the intention to cross-validate congruence relationships using LPA. This might represent a more comprehensive attempt to reveal complexities in disease state definition by patient and physicians that have eluded empirical investigation due to the use of difference scores.

Several limitations of our study should be acknowledged: first, LPA was used as an exploratory approach in our study; therefore, replication of our findings in independent samples is necessary. Although we considered validation in an independent sample within our patient population, this was not feasible; despite the absence of a formal approach in the literature for the definition of sample size requirements for the performance of LPA, a size of at least 500 seems to be a general consensus for best practice. ${ }^{44}$ Our cohort, representing a single centre, is not large enough to generate the requisite sample size for a separate validation set. It is encouraging, however, that a recent large study from the Early Rheumatoid Arthritis Network and British Society of Rheumatology Biologics Register revealed identical patient groupings to ours using a similar LPA approach. ${ }^{45}$ Second, since findings were based on a sample of Hispanic whites from the USA, our results may not be generalisable to other RA patient populations. Third, given the cross-sectional nature of our study, caution is recommended in interpreting the predictive power of the latent profiles as causal relationships cannot be inferred. Future research should examine the stability and longitudinal trajectories of latent profiles and their associations with clinical, functional and HRQoL outcomes. Fourth, physicians were blinded to PtGA scores and all patient-reported outcomes at the time of their MDGA assessment in our study; although this could theoretically impact the results, it is consistent with clinical trial practices and allows for a more impartial physician rating.

\section{CONCLUSION}

An unbiased, empirically derived, patient-centred LPA approach to characterise the relationship between patient and physician assessments of disease activity identified distinct, homogeneous and clinically meaningful patient groups. The five-profile solution was quantitatively and qualitatively distinct from the traditional threshold-based discordance definition; it confirmed that the magnitude of disease activity consensually reported by patients and physicians is a pivotal determinant of functional outcomes and demonstrated greater predictive power 
for disease-related, clinical, functional and HRQoL outcomes. Further validation of our findings may corroborate its clinical relevance, validity and suitability characterising the relationship between PtGA and MDGA.

Acknowledgements The authors would like to thank all patients who participated in this research and acknowledge the contributions of Harbor-UCLA Medical Center Rheumatologists Benedict Chou and Gopika Miller, who participated in the clinical evaluation and assessment of participants in the study.

Contributors All authors were involved in the study design and/or collection, analysis and interpretation of the data, provided critical revision of the manuscript and approved the final version to be submitted for publication.

Funding The authors have not declared a specific grant for this research from any funding agency in the public, commercial or not-for-profit sectors.

Competing interests None declared.

Patient consent Not required.

Ethics approval John F Wolf Human Subjects committee, Los Angeles Biomedical Research Institute at Harbor-UCLA Medical Center.

Provenance and peer review Not commissioned; externally peer reviewed.

Data sharing statement № additional data are available.

Open access This is an Open access article distributed in accordance with the Creative Commons Attribution Non Commercial (CC BY-NC 4.0) license, which permits others to distribute, remix, adapt, build upon this work non-commercially, and license their derivative works on different terms, provided the original work is properly cited, appropriate credit is given, any changes made indicated, and the use is non-commercial. See: http://creativecommons.org/licenses/by-nc/4.0/.

\section{REFERENCES}

1. Studenic P, Radner H, Smolen JS, et al. Discrepancies between patients and physicians in their perceptions of rheumatoid arthritis disease activity. Arthritis Rheum 2012;64:2814-23.

2. Khan NA, Spencer HJ, Abda E, et al. Determinants of discordance in patients' and physicians' rating of rheumatoid arthritis disease activity. Arthritis Care Res 2012;64:206-14.

3. Barton JL, Imboden J, Graf J, et al. Patient-physician discordance in assessments of global disease severity in rheumatoid arthritis. Arthritis Care Res 2010;62:857-64.

4. Karpouzas GA, Ramadan SN, Cost CE, et al. Discordant patientphysician assessments of disease activity and its persistence adversely impact quality of life and work productivity in US Hispanics with rheumatoid arthritis. RMD Open 2017;3:e000551.

5. Desthieux C, Hermet A, Granger B, et al. Patient-physician discordance in global assessment in rheumatoid arthritis: a systematic literature review with meta-analysis. Arthritis Care Res 2016;68:1767-73.

6. Aletaha D, Ward MM, Machold KP, et al. Remission and active disease in rheumatoid arthritis: defining criteria for disease activity states. Arthritis Rheum 2005;52:2625-36.

7. Ferreira RJO, Duarte C, Ndosi M, et al. Suppressing inflammation in rheumatoid arthritis: does patient global assessment blur the target? a practice-based call for a paradigm change. Arthritis Care Res 2018;70:369-78.

8. Edwards JR. Alternatives to difference scores: Polynomial regression analysis and response surface methodology. In: Drasgow F, Schmitt NW, Advances in measurement and data analysis. San Francisco: Jossey-Bass, 2002:350-400.

9. Klarenbeek NB, Koevoets R, van der Heijde DM, et al. Association with joint damage and physical functioning of nine composite indices and the 2011 ACR/EULAR remission criteria in rheumatoid arthritis. Ann Rheum Dis 2011;70:1815-21.

10. Marsh HW, Lüdtke $O$, Trautwein $U$, et al. Classical latent profile analysis of academic self-concept dimensions: Synergy of personand variable-centered approaches to theoretical models of selfconcept. Structural Equation Modeling: A Multidisciplinary Journal 2009;16:191-225

11. Bergman LR, Magnusson D. A person-oriented approach in research on developmental psychopathology. Dev Psychopathol 1997;9:291-319.

12. Laursen BP, Hoff E. Person-centered and variable-centered approaches to longitudinal data. Merrill Palmer Q 2006;52:377-89.

13. Magnusson D. The logic and implications of a person-oriented approach. In: Cairns RB, Bergman LR, Kagan J, Methods and models for studying the individual. Thousand Oaks, CA: Sage, 1998:33-63.

14. Walrath CM, Petras H, Mandell DS, et al. Gender differences in patterns of risk factors among children receiving mental health services: latent class analyses. J Behav Health Serv Res 2004;31:297-311.

15. McCutcheon AL. A Latent class analysis of tolerance for nonconformity in the American public. Public Opin Q 1985;49:474-88.

16. Pastor DA, Barron KE, Miller BJ, et al. A latent profile analysis of college students' achievement goal orientation. Contemp Educ Psychol 2007;32:8-47.

17. Karpouzas GA, Dolatabadi S, Moran R, et al. Correlates and predictors of disability in vulnerable US Hispanics with rheumatoid arthritis. Arthritis Care Res 2012;64:1274-81.

18. Aletaha D, Neogi T, Silman AJ, et al. 2010 rheumatoid arthritis classification criteria: an American College of Rheumatology/ European League Against Rheumatism collaborative initiative. Ann Rheum Dis 2010;69:1580-8.

19. Felson DT, Smolen JS, Wells G, et al. American College of Rheumatology/European League Against Rheumatism provisional definition of remission in rheumatoid arthritis for clinical trials. Arthritis Rheum 2011;63:573-86.

20. Nikiphorou E, Radner H, Chatzidionysiou K, et al. Patient global assessment in measuring disease activity in rheumatoid arthritis: a review of the literature. Arthritis Res Ther 2016;18:251.

21. Wolfe F, Clauw DJ, Fitzcharles MA, et al. The American College of Rheumatology preliminary diagnostic criteria for fibromyalgia and measurement of symptom severity. Arthritis Care Res 2010;62:600-10.

22. Bruce B, Fries JF. The Stanford Health Assessment Questionnaire: a review of its history, issues, progress, and documentation. $J$ Rheumatol 2003;30:167-78.

23. Kroenke K, Spitzer RL, Williams JB. The PHQ-9: validity of a brie depression severity measure. J Gen Intern Med 2001;16:606-13.

24. Huang FY, Chung H, Kroenke K, et al. Using the Patient Health Questionnaire-9 to measure depression among racially and ethnically diverse primary care patients. J Gen Intern Med 2006;21:547-52.

25. Ware JE, Sherbourne CD. The MOS 36-item short-form health survey (SF-36). I. Conceptual framework and item selection. Med Care 1992;30:473-83.

26. Gawlicki MC, Reilly MC, Popielnicki A, et al. Linguistic validation of the US Spanish work productivity and activity impairment questionnaire, general health version. Value Health 2006;9:199-204.

27. Kline RB. Principles and practice of structural equation modeling. 2. New York: Guilford Press, 2005.

28. Nylund KL, Asparouhov T, Muthen BO. Deciding the number of classes in latent class analysis and growth mixture modeling. Structural Equation Modelling 2007;14:535-69.

29. McLachlan GJ. Finite Mixture Models. New York: John Wiley, 2000.

30. Benjamini Y, Hochberg Y. Controlling the false discovery rate: a practical and powerful approach to multiple testing. J R Stat Soc, Series B 1995;57:289-300.

31. Katz P. Fatigue in rheumatoid arthritis. Curr Rheumatol Rep 2017;19:25.

32. Grøn KL, Ornbjerg LM, Hetland ML, et al. The association of fatigue, comorbidity burden, disease activity, disability and gross domestic product in patients with rheumatoid arthritis. Results from 34 countries participating in the Quest-RA program. Clin Exp Rheumatol 2014;32:869-77.

33. Gossec L, Steinberg G, Rouanet S, et al. Fatigue in rheumatoid arthritis: quantitative findings on the efficacy of tocilizumab and on factors associated with fatigue. The French multicentre prospective PEPS Study. Clin Exp Rheumatol 2015;33:664-70.

34. Margaretten M, Julian L, Katz P, et al. Depression in patients with rheumatoid arthritis: description, causes and mechanisms. Int J Clin Rheumtol 2011;6:617-23.

35. Koop SM, ten Klooster PM, Vonkeman HE, et al. Neuropathiclike pain features and cross-sectional associations in rheumatoid arthritis. Arthritis Res Ther 2015;17:237.

36. Dubois Mendes SM, Lôbo Queiroz BL, Santana LV, et al. Impact of different levels of disease activity in painful profile and quality of life in people with rheumatoid arthritis. Brazilian Journal of Medicine and Human Health 2014;2:1.

37. Smolen JS, Strand V, Koenig AS, et al. Discordance between patient and physician assessments of global disease activity in rheumatoid arthritis and association with work productivity. Arthritis Res Ther 2016;18:114.

38. Kaneko $\mathrm{Y}$, Kuwana $\mathrm{M}$, Kondo $\mathrm{H}$, et al. Discordance in global assessments between patient and estimator in patients with newly diagnosed rheumatoid arthritis: associations with progressive 
joint destruction and functional impairment. $J$ Rheumatol 2014;41:1061-6.

39. Nicassio PM, Kay MA, Custodio MK, et al. An evaluation of a biopsychosocial framework for health-related quality of life and disability in rheumatoid arthritis. J Psychosom Res 2011;71:79-85.

40. Wolfe F, Michaud K. Resistance of rheumatoid arthritis patients to changing therapy: discordance between disease activity and patients' treatment choices. Arthritis Rheum 2007;56:2135-42.

41. Fraenkel L, Cunningham M. High disease activity may not be sufficient to escalate care. Arthritis Care Res 2014;66:197-203.

42. Bergman LR, Trost K. The person-oriented versus the variableoriented approach: are they complementary, opposites, or exploring different worlds? Merrill Palmer Q 2006;52:601-32.
43. Wolfe F, Michaud K, Busch RE, et al. Polysymptomatic distress in patients with rheumatoid arthritis: understanding disproportionate response and its spectrum. Arthritis Care Res 2014;66:1465-71.

44. Finch $\mathrm{WH}$, Bronk KC. Conducting confirmatory latent class analysis using M plus. Structural Equation Modeling: A Multidisciplinary Journal 2011;18:132-51.

45. McWilliams DF, Ferguson E, Young A, et al. Discordant inflammation and pain in early and established rheumatoid arthritis: Latent Class Analysis of Early Rheumatoid Arthritis Network and British Society for Rheumatology Biologics Register data. Arthritis Res Ther 2016;18:295. 